\title{
Variant innervation of the mylohyoid muscle by the lingual nerve
}

\author{
A.J. Cooper ${ }^{1}$, A. Sadr' ${ }^{2}$, L. Xu3 ${ }^{3}$, R.S. Tubbs ${ }^{3-9}$, J. Iwanaga ${ }^{4,5,10,11}$ \\ ${ }^{1}$ Department of Biology, University of St. Francis, Joliet, IL, United States \\ ${ }^{2}$ Department of Restorative Dentistry, University of Washington School of Dentistry, Seattle, WA, United States \\ ${ }^{3}$ Department of Structural and Cellular Biology, Tulane University School of Medicine, New Orleans, LA, United States \\ ${ }^{4}$ Department of Neurosurgery, Tulane Centre for Clinical Neurosciences, Tulane University School of Medicine, \\ New Orleans, LA, United States \\ ${ }^{5}$ Department of Neurology, Tulane Centre for Clinical Neurosciences, Tulane University School of Medicine, \\ New Orleans, LA, United States \\ ${ }^{6}$ Department of Anatomical Sciences, St. George's University, St. George's, Grenada, West Indies \\ ${ }^{7}$ Department of Surgery, Tulane University School of Medicine, New Orleans, LA, United States \\ ${ }^{8}$ Department of Neurosurgery and Ochsner Neuroscience Institute, Ochsner Health System, New Orleans, LA, \\ United States \\ 'University of Queensland, Brisbane, Australia \\ ${ }^{10}$ Dental and Oral Medical Centre, Kurume University School of Medicine, Kurume, Fukuoka, Japan \\ ${ }^{11}$ Division of Gross and Clinical Anatomy, Department of Anatomy, Kurume University School of Medicine, Kurume, \\ Fukuoka Japan
}

[Received: 20 September 2021; Accepted: 27 October 2021; Early publication date: 5 November 2021]

\begin{abstract}
The nerve to mylohyoid muscle supplies the mylohyoid and the anterior belly of the digastric muscles, with terminal sensory branches that might innervate the submental skin and mandibular teeth. The nerve to mylohyoid muscle typically originates from the posterior surface of the inferior alveolar nerve right before entering the mandibular foramen. In rare cases, the nerve to mylohyoid muscle arises from the lingual nerve. The variations of the nerve to mylohyoid muscle might have led to failure of an inferior alveolar nerve blockade. During the routine dissection of a cadaveric head, a rare case was identified where the nerve to mylohyoid muscle had origins from both the inferior alveolar and lingual nerves. This case is reviewed and salient literature reviewed. (Folia Morphol 2022; 81, 4: 1079-1081)
\end{abstract}

Key words: nerve to mylohyoid muscle, lingual nerve, inferior alveolar nerve, cadaver, anatomy

\section{INTRODUCTION}

Typically, the lingual nerve (LN) branches off the mandibular division of the trigeminal nerve and supplies sensory fibres to the mucosa of the anterior two-thirds of the tongue, the floor of the mouth, and the lingual gingiva of the mandible. It descends medially and anteriorly to the inferior alveolar nerve (IAN) in the pterygomandibular space $[1,3]$. The nerve to mylohyoid (NM) originates from the pos- terior surface of the IAN just above the mandibular foramen. The NM is generally considered a motor nerve that supplies the mylohyoid muscle (MHM) and the anterior belly of the digastric muscle. As a variation, the nerve can have terminal sensory branches that might innervate the submental skin or mandibular teeth [6].

Variations of the NM include the following: originating inside the mandibular canal, originating and

Address for correspondence: J. Iwanaga, DDS, PhD, Department of Neurosurgery, Tulane Centre for Clinical Neurosciences, Tulane University School of Medicine, 131 S. Robertson St. Suite 1300, New Orleans, LA 70112, USA, tel: 5049885565, fax: 5049885793, e-mail: iwanagajoeca@gmail.com

This article is available in open access under Creative Common Attribution-Non-Commercial-No Derivatives 4.0 International (CC BY-NC-ND 4.0) license, allowing to download articles and share them with others as long as they credit the authors and the publisher, but without permission to change them in any way or use them commercially. 


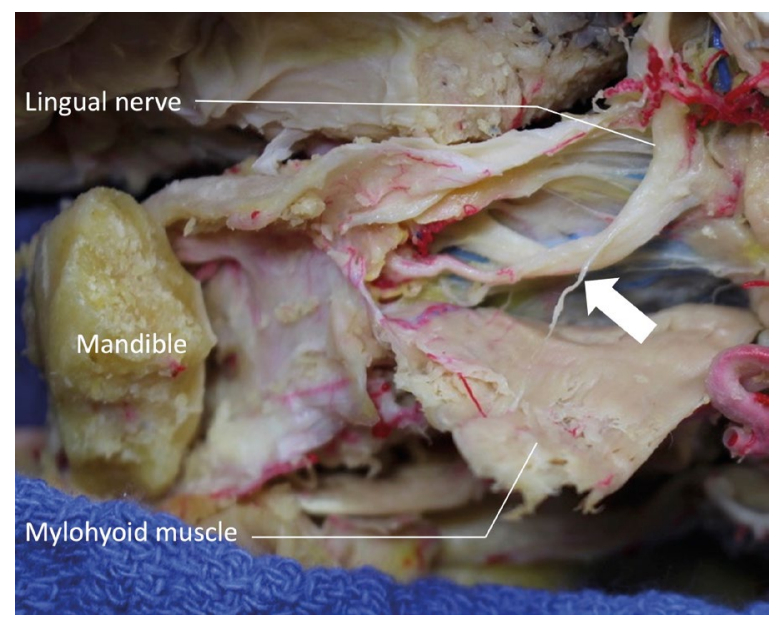

Figure 1. The variant nerve to the mylohyoid muscle (arrow) originating from the lingual nerve.

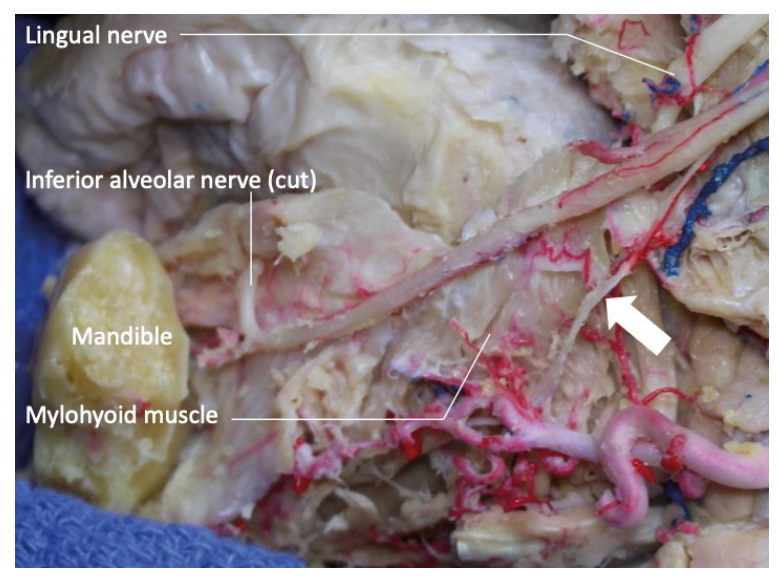

Figure 2. The normal nerve to the mylohyoid muscle (arrow) originating from the inferior alveolar nerve.

running parallel to the $L N$, running through bony grooves on the mandible, the presence of communication between the NM and LN near the submandibular ganglion and third molar tooth, and communication with the hypoglossal nerve to dually innervate the MHM [6, 7]. As a response to numerous of variations of the NM, an analysis of the anatomical characteristics of the NM, such as location of the starting point of the nerve, the distance from the nerve on the inferior border of the mandible to the menton and gonion, and the distribution of terminal branches have been made [6]. Due to the numerous variations of the NM, it is not surprising that IAN blockades can fail at providing full dental anaesthesia in mandibular surgeries [2]. In order to reduce such failure, it is important for dentists and oral surgeons to be aware of variations of the NM.

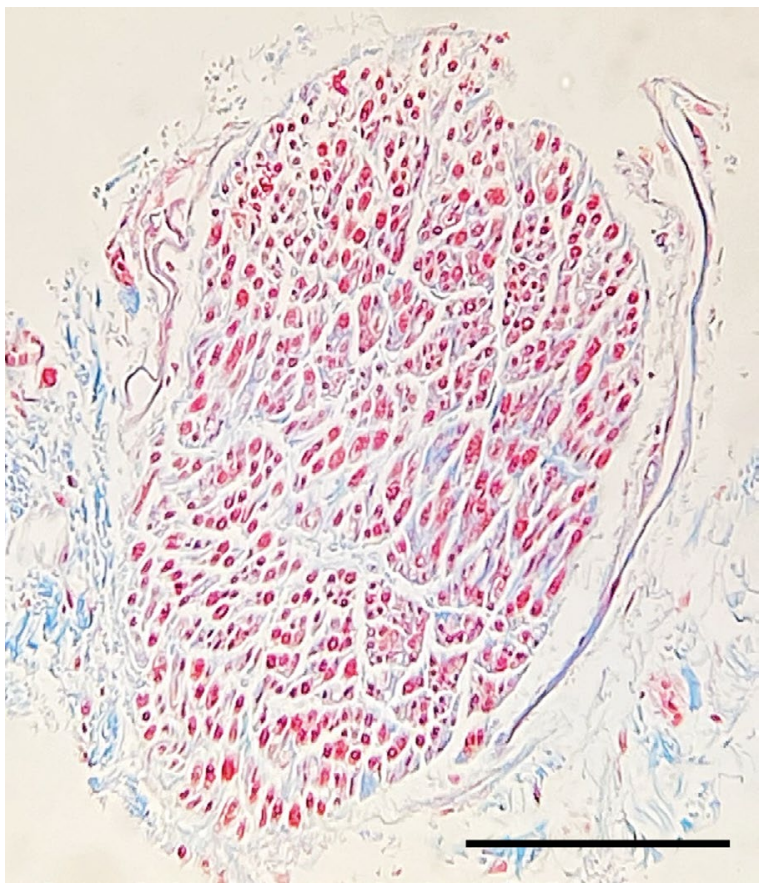

Figure 3. Harvested tissue consists of nerve fibres (Masson-trichrome staining). Scale bar: $50 \mu \mathrm{m}$.

Herein, we report a rare case where the MHM was innervated by both the IAN and LN.

\section{CASE REPORT}

During the routine dissection of an 82-year-old at death female cadaver, a variant innervation of the left MHM was found. A small branch $0.5 \mathrm{~mm}$ in diameter) arose from the lateral surface of the lingual nerve approximately $10 \mathrm{~mm}$ distal to the submandibular ganglion and terminated in the MHM (Fig. 1). The nerve ended at approximately $2 \mathrm{~cm}$ anterior to the posterior border of the MHM and did not pierce the muscle belly. Simultaneously, the muscle was also innervated by the normal NM that arose from the posterior surface of the inferior alveolar nerve and innervated the inferior (lateral) aspect of the muscle (Fig. 2). Histological observation (Masson-trichrome staining) revealed that the harvested tissue was nerve (Fig. 3). No variation was found in the same area on the right side. No surgical scars or other anatomical variations were found in the dissected area.

\section{DISCUSSION}

It has been reported that the richest nerve arborisation of the NM was in the middle one-third of the MHM. Terminal branches of the NM have also been 
reported to travel on the surface of the MHM and penetrate its surface [6]. Embryologically, the nerve of the first pharyngeal arch (trigeminal nerve) innervates the muscles of mastication, tensor tympani, tensor veli palatini, and MHM and anterior belly of the digastric. The MHM is one of the first pharyngeal arch muscles to differentiate. It initially attaches to the ventral border of Meckel's cartilage and later migrates, attaching to the foetal mandible [9].

Along with the several variations in the origin and branching patterns of the NM there have been cases reported on the connections between the LN and NM. In 1 case it was observed that there was an unusual dual innervation of the submandibular gland from both the LN and the NM [7]. The NM was found medial and slightly inferior to the submental artery and was found in the submandibular gland without piercing it. In this case, there was also a posterior branch to the submandibular gland from the LN, which had no communicating branch to the NM. This suggested that the submandibular gland might have been innervated by both sensory fibres from the LN and postganglionic parasympathetic fibres from the NM [7]. In another report, a rare occurrence where the NM originated from the LN near the submandibular duct and pierced the MHM to innervate the anterior belly of digastric and MHM was found [3]. The variant NM in the case reported herein might have resulted from nerve fibres from the mandibular nerve "hitchhiking" along the $L N$ in the infratemporal fossa instead of joining the IAN.

Studies have shown that in $43-50 \%$ of the population, the NM innervates the mandibular incisors and related gingivae. In rare cases, the NM and LN were observed to have communication in the submandibular triangles, and proximal to the lower third molar tooth [8]. Other reports have shown the NM originating from the glossopharyngeal nerve or the $L N$ and communicating with the IAN. Also, the NM may travel through other foramina in the mandible, which may be another reason for failed IAN blocks since the NM does not consistently originate from the IAN [10]. Since the origin of the NM varies, successfully finding its location has proved difficult in various procedures.

An IAN block will only be effective in mandibular surgical procedures when it is delivered correctly and the NM arises from the IAN. Therefore, variation of the location of the NM and its complex origin may contribute to the failure of IAN blocks. Although the regular course of the NM has been well documented $[4,6]$, further research on variant pathways of the NM is important because it allows dentists and oral surgeons to better assess procedural risks and better understand complications.

\section{CONCLUSIONS}

A rare case of MHM innervation is reported. Such cases are important for dentists and oral surgeons to consider, especially during mandibular surgeries.

\section{Acknowledgements}

The authors sincerely thank those who donated their bodies to science so that anatomical research could be performed. Results from such research can potentially increase mankind's overall knowledge that can then improve patient care. Therefore, these donors and their families deserve our highest gratitude [5].

\section{Conflict of interest: None declared}

\section{REFERENCES}

1. Behnia H, Kheradvar A, Shahrokhi M. An anatomic study of the lingual nerve. J Oral Maxillofac Surg. 2000; 58: 649-651, doi: 10.1053/jo.2000.6209.

2. Clark S, Reader A, Beck M, et al. Anesthetic efficacy of the mylohyoid nerve block and combination inferior alveolar nerve block/mylohyoid nerve block. Oral Surg Oral Med Oral Pathol Oral Radiol Endod. 1999; 87(5): 557-563, doi: 10.1016/s1079-2104(99)70133-2, indexed in Pubmed: 10348512.

3. Iwanaga J, Kikuta S, Oskouian RJ, et al. Nerve to mylohyoid branched from the lingual nerve: previously undescribed case. Anat Sci Int. 2019; 94(3): 266-268, doi: 10.1007/ s12565-019-00476-4, indexed in Pubmed: 30710312.

4. Iwanaga J, Kim HJ, Wysiadecki G, et al. Localizing the nerve to the mylohyoid using the mylohyoid triangle. Anat Cell Biol. 2021; 54(3): 304-307, doi: 10.5115/acb.21.019, indexed in Pubmed: 33941711.

5. Iwanaga J, Singh V, Ohtsuka A, et al. Acknowledging the use of human cadaveric tissues in research papers: Recommendations from anatomical journal editors. Clin Anat. 2021; 34(1): 2-4, doi: 10.1002/ca.23671, indexed in Pubmed: 32808702.

6. Ryu EJ, Kim DH. Anatomical insights of the mylohyoid for clinical procedures in dentistry. Clin Anat. 2021; 34(3): 461-469, doi: 10.1002/ca.23675, indexed in Pubmed: 32893917.

7. Ryumon $S$, Hage D, lbaragi $S$, et al. Dual innervation of the submandibular gland by nerve to mylohyoid and chorda tympani. Morphologie. 2021; 105(351): 316-318, doi: 10.1016/j. morpho.2020.11.004, indexed in Pubmed: 33288421.

8. Sato I, Sunohara M, Ueno R, et al. Branch of mylohyoid and lingual nerves on submandibular and submental triangles. Okajimas Folia Anat Jpn. 2004; 81(2-3): 45-48, doi: 10.2535/ofaj.81.45, indexed in Pubmed: 15455728.

9. Sperber GH, Sperber SM, Guttmann GD. Craniofacial embryogenetics and development, 3rd edn. People's Medical Publishing House, Beijing 2018.

10. Tubbs RS, Shoja MM, Loukas M. Bergman's comprehensive encyclopedia of human anatomic variation. John Wiley \& Sons, Hoboken, NJ 2016. 